\title{
Corrigendum: Genome-wide association analyses in Han Chinese identify two new susceptibility loci for amyotrophic lateral sclerosis
}

Min Deng, Ling Wei, Xianbo Zuo, Yanghua Tian, Fei Xie, Panpan Hu, Chunyan Zhu, Fengqiong Yu, Yu Meng, Honghao Wang, Fangfang Zhang, Huijuan Ma, Rong Ye, Huaidong Cheng, Jing Du, Wenwen Dong, Shanshan Zhou, Changqing Wang, Yu Wang, Jingye Wang, Xianwen Chen, Zhongwu Sun, Nong Zhou, Yubao Jiang, Xiuxiu Liu, Xiaogang Li, Nan Zhang, Na Liu, Yingjun Guan, Yongsheng Han, Yongzhu Han, Xinyi Lv, Yu Fu, Hui Yu, Chunhua Xi, Dandan Xie, Qiyuan Zhao, Peng Xie, Xin Wang, Zhijun Zhang, Lu Shen, Yong Cui, Xianyong Yin, Hui Cheng, Bo Liang, Xiaodong Zheng, Tatia M C Lee, Gang Chen, Fusheng Zhou, Jan H Veldink, Wim Robberecht, John E Landers, Peter M Andersen, Ammar Al-Chalabi, Chris Shaw, Chunfeng Liu, Beisha Tang, Shangxi Xiao, Janice Robertson, Fengyu Zhang, Leonard H van den Berg, Liangdan Sun, Jianjun Liu, Sen Yang, Xiaodong Ju, Kai Wang \& Xuejun Zhang Nat. Genet.; doi:10.1038/ng.2627; published online 28 April 2013; corrected online 9 May 2013

In the version of this article initially published online, the affiliations of authors Xiuxiu Liu, Xiaogang Li, Nan Zhang and Na Liu were incorrect. The correct affiliation of these authors is the Department of Neurology, Peking University Third Hospital, Beijing, China. The errors have been corrected in the PDF, HTML and print versions of this article. 